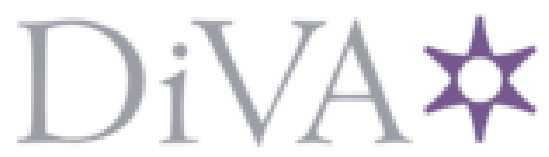

http://www.diva-portal.org

\title{
Postprint
}

This is the accepted version of a paper published in IEEE signal processing magazine (Print). This paper has been peer-reviewed but does not include the final publisher proof-corrections or journal pagination.

Citation for the original published paper (version of record):

Nilsson, J., Händel, P. (2013)

Fast Argument Quantization.

IEEE signal processing magazine (Print), 30(6): 169-172

http://dx.doi.org/10.1109/MSP.2013.2276531

Access to the published version may require subscription.

N.B. When citing this work, cite the original published paper.

Permanent link to this version:

http://urn.kb.se/resolve?urn=urn:nbn:se:kth:diva- 133476 


\title{
Fast Argument Quantization
}

\author{
John-Olof Nilsson and Peter Händel
}

A quantized low-resolution argument of a complex number or twodimensional vector is required in many digital signal processing algorithms. Examples include: amplitude and phase-shift keying (APSK) code demodulation for which it may be used to evaluate the Voronoi diagram; low-level processing for many computer vision methods which exploit histograms of gradient sample arguments, e.g. scale-invariant-feature-transform (SIFT) and histogram-of-orientedgradients (HOG); and phase tracking/frequency estimation for which it may be used as a low-cost phase approximation. Often, such quantized arguments will have to be computed a large number of times and under real-time constraints. Therefore, efficient solutions to these calculations are of interest.

The argument quantization can be viewed as two-dimensional data binning in equidistant sectors as illustrated in Figure 1. Consequently, we present three efficient solutions to this data binning problem. First, a straight-forward solution explicitly calculating the complex/vector argument is presented. Secondly, we present a solution based on projection and bisection which can be implemented without any division and with only integer data representation. Thirdly, we present a phase rotation based solution suitable for vectorization/parallelization. The solutions we refer to as binning by argument (BBA), binning by projection (BBP), and binning by rotation (BBR). We also present ways of finding the first 3 bits, i.e. the half-plane, quad- rant, and octant sector, using bitmanipulations. Finally, a table of types and numbers of required operations for the implementation of each solution is presented. Further details and extensions of the presented methods can be found in the report [1].

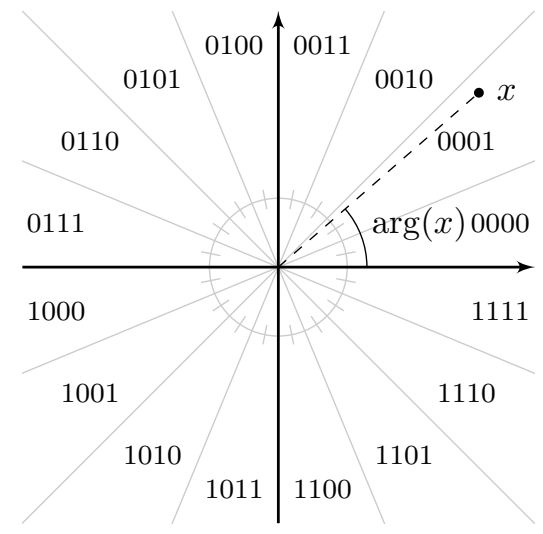

Fig. 1: Sample $x$ falls in sector 1 .

\section{Sector binning}

The data binning in sectors, or sector binning, entails finding the sector that a complex number $x=$ $x_{0}+j x_{1}$ or two-dimensional vector $x=\left(x_{0}, x_{1}\right)$ occupies. The problem is illustrated in Figure 1 for 16 equidistant sector bins, corresponding to a 4-bit argument quantization. Formally, the sector binning can be described by the mapping

$$
\begin{gathered}
g: x \mapsto n \\
\text { s.t. } 2 \pi \frac{n}{N} \leq \arg (x)<2 \pi \frac{n+1}{N}
\end{gathered}
$$

where $n$ is the sector bin number, $N$ is the total number of sectors, $\arg (\cdot)$ is the complex/vector argument defined on $[0,2 \pi)$, and $2 \pi \frac{n}{N}$ and $2 \pi \frac{n+1}{N}$ are the delimiting arguments of the sector $n$. The sector binning (1) may also be extended to the non-equidistant case with arbitrary delimiting arguments [1].

\section{Binning by argument}

The straight-forward way of implementing the sector binning, as described by (1), is by explicitly calculating the argument $\arg (x)$. By simple manipulations, (1) yields

$$
n=\left\lfloor\frac{N}{2 \pi} \arg (x)\right\rfloor
$$

where $\lfloor\cdot\rfloor$ denotes the flooroperation. Consequently, by calculating $\arg (x)$, the sector $n$ can easily be found. Now, $\arg (x)=$ $\operatorname{atan} 2\left(x_{1}, x_{0}\right)$, which is $\operatorname{atan}\left(\left|\frac{x_{1}}{x_{0}}\right|\right)$ mapped onto the four quadrants. Unfortunately, the evaluation of $\operatorname{atan}(\cdot)$ can incur a significant computational cost.

Multiple fast approximations of $\operatorname{atan}(\cdot)$ and $\operatorname{atan} 2(\cdot, \cdot)$ are available in the literature [2][3] and have previously been presented in this column [4][5]. For the sector binning we suggest using an $\operatorname{atan} 2(\cdot, \cdot)$ implementation based on the $\operatorname{atan}(\cdot)$ approximation number 7 from [5],

$$
\operatorname{atan}(z) \approx \frac{\pi}{4} z+0.273 z(1-|z|) .
$$

Together with the relation $[6]$

$$
\operatorname{atan}\left(\left|\frac{x_{1}}{x_{0}}\right|\right)=\frac{\pi}{4}+\operatorname{atan}(z)
$$

where

$$
z=\frac{\left|x_{1}\right|-\left|x_{0}\right|}{\left|x_{1}\right|+\left|x_{0}\right|}
$$

it gives

$$
\begin{aligned}
& \frac{N}{2 \pi} \operatorname{atan}\left(\left|\frac{x_{1}}{x_{0}}\right|\right) \approx \\
& \frac{N}{8}+\frac{N}{8} z+\frac{0.273 N}{2 \pi} z(1-|z|) .
\end{aligned}
$$

This approximation lets us exploit the factor $\frac{N}{2 \pi}$ to simplify the implementation and has an accuracy to computational cost tradeoff suitable for up to some hundred equidistant sector bins (maximum error corresponding to $0.22^{\circ}$ ). 
The approximation (3) gives the scaled argument of $x$ in the first quadrant. Now, we want to extend it to all four quadrants. This can be done with a series of bit manipulations and an addition. Let the symbols $\oplus$ and $\wedge$ denote the logical XOR and AND operations, respectively. Let the single bit $s$ indicate the even/odd status of the quadrant of $x$, i.e.

$$
s=\operatorname{sb}\left(x_{0}\right) \oplus \operatorname{sb}\left(x_{1}\right)
$$

where $\operatorname{sb}(\cdot)$ denotes the sign-bit (1 if the number is negative and 0 if it is positive). Let $r$ be an integer number with the first bit equal to $\operatorname{sb}\left(x_{0}\right)$, the second bit equal to $\operatorname{sb}\left(x_{1}\right) \wedge s$, and the remaining bits zero (i.e. $r=0,1,2$ ). Further, define the sign-bit-copy function

$$
\operatorname{sbc}(\alpha, \beta)= \begin{cases}\beta & \text { if } \alpha=0, \\ -\beta & \text { if } \alpha=1,\end{cases}
$$

where $\alpha$ is a single bit and $\beta$ is a positive number. Then (3) can be mapped onto the four quadrants by

$$
\begin{aligned}
& \frac{N}{2 \pi} \operatorname{atan} 2\left(x_{1}, x_{0}\right)= \\
& \quad \frac{N}{2} r+\operatorname{sbc}\left(s, \frac{N}{2 \pi} \operatorname{atan}\left(\left|\frac{x_{1}}{x_{0}}\right|\right)\right) .
\end{aligned}
$$

Apart from bit-manipulations, this requires 1 division, 2-3 multiplications, and 6 additions; and no conditional branches are required. In case $N$ is a power of 2 , the multiplications with $\frac{N}{2}$ and $\frac{N}{8}$ can be implemented with left shifts. In case $N$ is not a power of $2, \frac{N}{2} r$ can preferably be implemented with a memory access by $r$ in the 3 element look-up table $\left[0, \frac{N}{2}, N\right]$.

With this approximation of $\frac{N}{2 \pi} \operatorname{atan} 2\left(x_{1}, x_{0}\right)$, the relation (2) gives the sector bin $n$. We refer to this as binning by argument or BBA. While simple and straight-forward, this solution to the sector binning unfortunately has a number of undesirable properties. First of all, it requires a division operation, which is slow on many general purpose processing platforms and requires a large number of gates if implemented in hardware. Secondly, it requires non-integer data types, which obviously complicates the implementation and adds numerical approximation issues. Thirdly, it exploits the function approximation (3), which gives systematic errors in the binning, i.e. the de facto delimiting arguments will be slightly off from those in (1). (See [5] for a detailed error plot.) These properties hold for any implementation in which the argument is explicitly calculated. Therefore, for many platforms and applications, alternatives are desirable.

\section{Binning by projection}

Implementing the sector binning by computing the argument might seem a little backward. A highly accurate argument is calculated while all we need to know is between which two lines in Figure 1 that $x$ falls. Obviously, the argument is not needed to find that out.

If $p$ is a vector, such that $\arg (p)=2 \pi \frac{n}{N}$ and $q$ is a vector rotated counter clock-wise by $\pi / 2$ relative $p$. Then, as shown for two samples $x$ and $x^{\prime}$ in Figure 2, if the argument difference between a sample and $p$ lies between $\pi$ and $-\pi$, the sign (bit) of the projection of the sample on $q$ can be used to determine whether its argument is smaller or larger than the argument $2 \pi \frac{n}{N}$ of $p$.

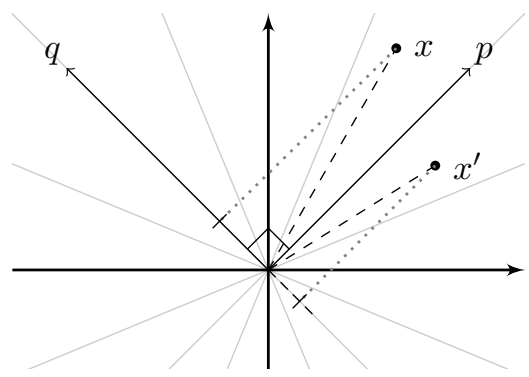

Fig. 2: Projection of $x$ and $x^{\prime}$ on $q$.

The sign of the projection is the same as the sign of the scalar product between $x$ and $q$. By defining vectors $q_{n}$ such that

$$
\arg \left(q_{n}\right) \stackrel{2 \pi}{\equiv} 2 \pi \frac{n}{N}+\frac{1}{2} \pi
$$

where $\stackrel{2 \pi}{=}$ denotes equality modulus $2 \pi$, the sector binning (1) can equiv- alently be written

$$
\begin{gathered}
g: x \mapsto n \\
\text { s.t. } \quad x \cdot q_{n} \geq 0>x \cdot q_{n+1}, \\
-\pi<2 \pi \frac{n}{N}-\arg (x) \leq \pi, \\
-\pi<2 \pi \frac{n+1}{N}-\arg (x) \leq \pi
\end{gathered}
$$

where - denote the scalar product. This projection approach partially resembles/generalizes the ideas presented in [7][8].

If $N$ is a power $\mathrm{M}$ of $2\left(N=2^{M}\right)$, then (5) is easily solved by bisection. Let $n$ be an integer variable initialized to 0 and let a leading superscript $^{i}(\cdot)$ indicate the $i$ th bit of the variable. Then the loop

$$
\begin{aligned}
\text { loop } i & =M-1: 1 \\
{ }^{i} n & =1 \\
d & =x \cdot q_{n} \\
{ }^{i} n & ={ }^{i} n \oplus \operatorname{sb}(d)
\end{aligned}
$$

gives the solution to the sector binning (5). This is what we refer to as binning by projection or BBP. Note that here $=$ has been used to denote assignment and that the loop goes from higher to lower values of $i$. The projections (inner products) in the loop is illustrated in Figure 3 where $q_{n}$ of the $\ell$ th loop is denoted with $q_{n_{\ell}}$. Each projection on $q_{n_{\ell}}$ successively halves the search space (with middle $\left.\arg \left(q_{n_{\ell}}\right)-\pi / 2\right)$ of sectors in which $x$ can be found. The BBP solution can also be extended to the case when $N$ is not a power of 2 and the sector bins are not equidistant [1].

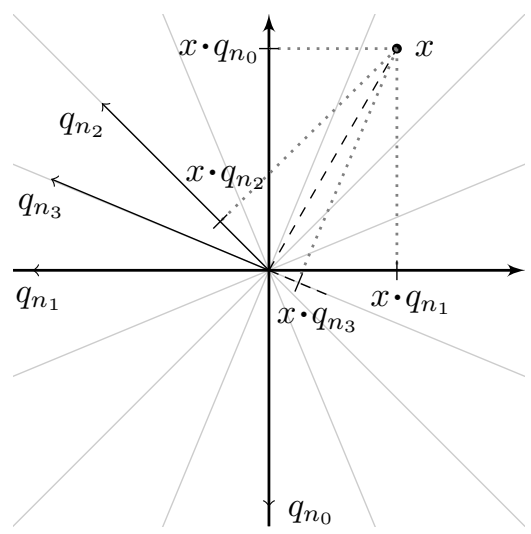

Fig. 3: Projections of $x$ on $q_{n_{\ell}}$.

The beauty of the projection and bisection is that we may choose 
$q_{n}$ arbitrarily, as long as (4) is satisfied. Therefore, $q_{n}$ may be chosen such that the implementation of the scalar product $x \cdot q_{n}$ is simplified. This means that one or both of the multiplications in $x \cdot q_{n}$ can be avoided. Also, the solution only requires additions, multiplications, and bit-manipulations. Therefore, no non-integer data types are required. Obviously BBP does not suffer from the undesirable properties of BBA. However, instead it has a computational cost which grows as the logarithm of $N(M-1$ loop iterations) and the bisection requires data dependent memory accesses in a small $q_{n}$ look-up table. The latter can be undesirable since the memory accesses may be slow when compared to arithmetic operations and they cannot easily be vectorized.

\section{Binning by rotation}

In case data dependent memory accesses are undesirable, the sector binning can be solved in a recursive manner without any memory accesses by manipulating $x$ itself. Treating $x$ as a complex number, means its argument can be manipulated by multiplying it with another complex number. This will rotate $x$ with the argument of the complex number. Rotating $x$ by $\pi$ and the sign of $x_{1}$ will tell us in which half plane $x$ is in. Rotating the product with $\pi / 2$ towards zero argument will then result in the sign of $x_{1}$ giving us the quadrant of $x$. Continuing rotating with $\pi / 4$ gives the octant and so forth. Along these lines and with the rotating complex numbers $c_{i}$, with real parts $a_{i}$ and imaginary parts $b_{i}$, chosen such that

$$
\arg \left(c_{i}\right)=\pi 2^{-i+(M-1)} ;
$$

i.e. $\arg \left(c_{M-1}\right)=\pi, \arg \left(c_{M-2}\right)=$ $\pi / 2, \arg \left(c_{M-3}\right)=\pi / 4$, and so forth; the sector bin number $n$ (initialized to 0 ) of $x$ can recursively be calculated with

$$
\begin{aligned}
\text { loop } i & =M-1: 1 \\
{ }^{i} n & =1 \\
b_{i} & =-\operatorname{sign}\left(x_{1}\right) b_{i} \\
x & =x \times c_{i} \\
{ }^{i} n & ={ }^{i} n \oplus \operatorname{sb}\left(x_{1}\right)
\end{aligned}
$$

where $\times$ denotes the complex multiplication. We refer to this as binning by rotation or BBR. The switching of the sign of $b_{i}$ ensures that the rotation of $x$ is always towards zero argument. Note that $-\operatorname{sign}\left(x_{1}\right) b_{i}$ can be implemented with $\operatorname{sbc}\left(\neg \operatorname{sb}\left(x_{1}\right), b_{i}\right)$, where $\neg$ denotes the NOT operation. Consequently, it only requires bitmanipulations. The manipulation of $x$ by the loop is illustrated in Figure 4 where $c_{i_{\ell}}$ is used to denote the rotating complex number of the $\ell$ th loop.

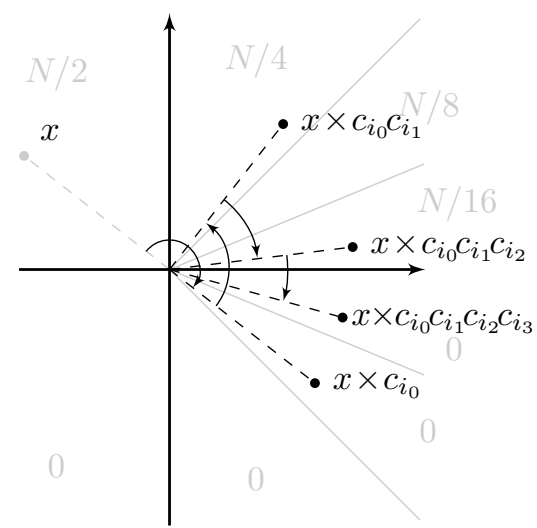

Fig. 4: Rotations of $x$ by $c_{i}$.

Each rotation halves the number of potential sectors and therefore, the loop in essence describes another form of bisection. The number of required arithmetic operations for evaluating (8) is larger when compared to (6). However, the data dependent memory accesses have been avoided. Unfortunately, this solution cannot be extended to non-equidistant and non-power-of2 number of sector bins. Similar to the choices of $q_{n}$ in BBP, the primitive rotations $c_{i}$ can be chosen to simplify the implementation of (8), letting us avoid multiplication operations in $x \times c_{i}$. Also, only bitmanipulations, additions, and multiplications are required; and therefore, integer data types can be used throughout.

Finding half-plane, quadrant, and octant

The first three loops in (6) correspond to finding the half-plane, quadrant, and octant sector which $x$ occupies. These first three loops can be simplified. The first loop/bit (the half plane) is simply given by the sign bit of $x_{1}$

$$
{ }^{M-1} n=\operatorname{sb}\left(x_{1}\right) .
$$

The second loop/bit (the quadrant) is given by

$$
{ }^{M-2} n={ }^{M-1} n \oplus \operatorname{sb}\left(x_{0}\right) .
$$

Finally, the third loop/bit (octant) is given by

${ }^{M-3} n={ }^{M-2} n \oplus \operatorname{sb}\left(\left|x_{0}\right|-\left|x_{1}\right|\right)$.

For subsequent loops, no straightforward simplifications compared to (6) can be found. Using (9)-(11), instead of the first three bisection loops, will significantly reduce the cost of the bisection. Also, for applications in which binning in only 4 or 8 sectors is desired $(M=2$ and $M=3$ ), this will directly give the sector bin number without any multiplications.

Expression (9) and (10) can also be used to replace the first two loops of (8). However, for (8), $x$ needs to be rotated accordingly which can be achieved with the initial reassignment

$$
\left[\begin{array}{l}
x_{0} \\
x_{1}
\end{array}\right]=\left[\begin{array}{c}
\left|x_{1}\right| \\
-\operatorname{sign}\left(x_{1}\right) x_{0}
\end{array}\right] .
$$

\section{Computational cost}

Apart from the atan( $\cdot$ ) approximation and the non-integer variable and constant representations in BBA and the necessary numerical approximations in the relations (4) and (7) when selecting $q_{n}$ and $c_{i}$ for $\mathrm{BBP}$ and $\mathrm{BBR}$, respectively, all three solutions, BBA, $\mathrm{BBP}$, and BBR, have the same logical result. Therefore, their performance is primarily determined by their computational requirements and costs.

The exact numbers and types of required operations for the different solutions are dependent on many implementation choices. However, in Table 1, a rough picture of the approximative numbers and types of operations required for $\mathrm{BBA}, \mathrm{BBP}$, and BBR is given. The operations have been divided into: additions and subtractions (add/sub), multiplications (mul), divisions (div), absolute values (abs), memory accesses (mov), bitshifts (shift), bit-assignments (bitassign), bit-wise XOR/AND/NOT 
(bit-op), and other miscellaneous operations/functions (other). Further, it has been assumed that the number of sector bins $N$ is $2^{M}$ (a power of 2); the half-plane, quadrant, and octant sector calculations are used; and for both BBP and BBR, one and two multiplications in $x=x \cdot q_{i}$ and $x=x \times c_{i}$, respectively, are replaced with bit-shifting and signcopy. Finally, the loops are assumed unrolled, and therefore, no operations for iteration variable manipulation are counted.

\section{Discussion and conclusions}

We have presented three different solutions to sector binning of a complex number or two-dimensional vector. This binning can be viewed as a quantization of the related argument. Table 1 shows the strengthes and weaknesses of each solution. BBA requires division and non-integer data types, but the computational cost is independent of the number of sector bins. BBP requires the least number of operations for a small number of sectors, but the computational cost scales with the logarithm of $N$ and data dependent memory accesses are required. BBR avoids the data dependent memory accesses at the cost of a somewhat higher number of required operations. Both BBP and BBR can be implemented with only integer data types.

Which solution to favor is a matter of the intended platform, the number of sector bins/quantization level, and the application. For embedded platforms, a moderate number of bins, and sequential processing typical for communication applications, we would generally favor BBP. However, for processing of large data chunks (e.g. images), opting for vectorization, and for platforms with readily available multipliers, we would favor BBR. Finally, for platforms with a fast division operation and in the case of a large non-power-of- 2 number of sector bins or floating-point data, we would favor BBA.

\section{Authors}

Ph.D candidate John-Olof Nilsson and Prof. Peter Händel are with the Signal Processing Lab, ACCESS Linnaeus Centre, KTH Royal Institute of Technology, Stockholm, Sweden.

\section{References}

[1] J.-O. Nilsson, "Efficient implementation of data binning in sectors," Tech. Rep. TRITA-EE 2012:041, School of Electrical Engineering, KTH Royal Institute of Technology, 2012.

[2] J. F. Hart, Computer approximations. Wiley, 1968.

[3] J. Crenshaw, Math Toolkit for Real-Time Programming. CMP, 2000.

[4] R. Lyons, "Another contender in the arctangent race," Signal Processing Magazine, IEEE, vol. 21, pp. 109 - 110, Jan 2004.

[5] S. Rajan, S. Wang, R. Inkol, and A. Joyal, "Efficient approximations for the arctangent function," Signal Processing Magazine, IEEE, vol. 23, pp. 108 -111, May 2006.

[6] S. Rajan, S. Wang, and R. Inkol, "Efficient approximations for the four-quadrant arctangent function," in Proc. CCECE'06, pp. 1043 -1046, May 2006.

[7] T. P. Cao and G. Deng, "Realtime vision-based stop sign detection system on FPGA," in Proc. DICTA'08, 2008.

[8] S. Bauer, U. Brunsmann, and S. Schlotterbeck-Macht, "FPGA Implementation of a HOG-based Pedestrian Recognition System," in Proc. MPC-Workshop, July 2009.

\begin{tabular}{|r|c|c|c|}
\hline & BBA & BBP & BBR \\
\hline add/sub & 6 & $(M-3)+1$ & $2(M-2)$ \\
\hline mul & 2 & $(M-3)$ & $2(M-2)$ \\
\hline div & 1 & 0 & 0 \\
\hline abs & 3 & 2 & 0 \\
\hline mov & 0 & $3(M-3)$ & 0 \\
\hline shift & 2 & $(M-3)$ & $2(M-2)$ \\
\hline bit-assign & 3 & $2(M-3)+3$ & $2(M-2)+2$ \\
\hline bit-op & 2 & $(M-3)+2$ & $(M-2)+2$ \\
\hline \multirow{2}{*}{ other } & $\begin{array}{c}\text { floor, } \\
\text { sbc }(\cdot)\end{array}$ & $M$ signcopy & sbc $(\cdot)$, \\
& $2 M$ signcopy \\
\hline
\end{tabular}

Table 1: Operations required to perform the sector binning. 